\title{
Desempenho de um trator agrícola em operações de preparo de solo no estado de Mato Grosso
}

\author{
Diego Augusto FIORESE ${ }^{1 *}$, Thiago Martins MACHADO ${ }^{1}$, Rodrigo Sinaidi ZANDONADI ${ }^{1}$, \\ Renan Felipe Almeida de ARAÚJO ${ }^{1}$, Gabriel Felipe da Silva RICARDO ${ }^{1}$, Janaina da Silva SCHIMITT ${ }^{1}$ \\ ${ }^{1}$ Instituto de Ciências Agrárias e Ambientais, Universidade Federal de Mato Grosso, Sinop, MT, Brasil. \\ E-mail: dafiorese@yahoo.com.br
}

Recebido em outubro/2018; Aceito em fevereiro/2019.

RESUMO: Objetivou-se avaliar o desempenho de um trator de $134 \mathrm{~kW}$ com quatro equipamentos de preparo do

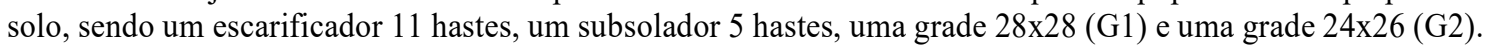
$\mathrm{O}$ delineamento foi em blocos casualizados com esquema fatorial, onde avaliou-se principalmente a demanda de tração e o consumo de combustível em duas marchas de trabalho compatíveis para cada operação. As máximas demandas de tração foram: 44,9 kN (escarificação); 38,1 kN (subsolagem); 31,1 kN (G1); e 24,5 kN (G2). Os consumos operacionais não tiveram variação entre as marchas $\left(4,5\right.$ e $\left.6 \mathrm{~km} \mathrm{~h}^{-1}\right)$ para a escarificação e subsolagem, com médias de 16,9 a 23,5 $\mathrm{L} \mathrm{ha}^{-1}$, respectivamente. Com as grades, houve variação de 1,5 a $2 \mathrm{~L} \mathrm{ha}^{-1}$ de economia quando operando em marchas mais rápidas, tendo-se o menor consumo de $12,9 \mathrm{~L} \mathrm{ha}^{-1}\left(\mathrm{G} 1 \mathrm{a} 7,3 \mathrm{~km} \mathrm{~h}^{-1}\right)$ e $11,6 \mathrm{~L}$ ha ${ }^{-1}$ (G2 a 9,2 $\left.\mathrm{km} \mathrm{h}^{-1}\right)$. Conclui-se que para mobilização do solo a até $0,3 \mathrm{~m}$ de profundidade, a escarificação é mais vantajosa que a subsolagem, promovendo economia de $6,6 \mathrm{~L} \mathrm{ha}^{-1}$. Recomenda-se utilizar as marchas de maior velocidade, dentro do permitido para o tamanho do trator e para a qualidade da operação.

Palavras-chave: força de tração, consumo de combustível, escarificação, subsolagem, gradagem.

\section{Agricultural tractor performance in soil tillage operations in the state of Mato Grosso - Brazil}

\begin{abstract}
The objective of this work was to evaluate the performance of a $134 \mathrm{~kW}$ tractor with four tillage equipment, with a 11 shank light subsoiler (for scarification), a 5 shank heavy subsoiler, a Offset disk harrow $28 \times 28$ (G1) and a Offset disk harrow 24x26 (G2). The experimental design was in randomized blocks in factorial scheme, where traction demand and fuel consumption was evaluated in two ground speed for each operation. The maximum drawbar pull was: $44.9 \mathrm{kN}$ (scarification); $38.1 \mathrm{kN}$ (subsoiling); $31.1 \mathrm{kN}$ (G1); and 24.5 kN (G2); The operational consumption did not vary between the ground speed allowed by the different gears (4.5 and 6 $\mathrm{km} \mathrm{h}^{-1}$ ) for scarification and subsoiling, varying from 16.9 and $23.5 \mathrm{~L} \mathrm{ha}^{-1}$, respectively. With the disk harrows, there was a variation of 1.5 to $2 \mathrm{~L} \mathrm{ha}^{-1}$ of fuel savings when operating at higher gear, with the lowest consumption of $12.9 \mathrm{~L} \mathrm{ha}^{-1}\left(\mathrm{G} 1\right.$ at $\left.7.3 \mathrm{~km} \mathrm{~h}^{-1}\right)$ and $11.6 \mathrm{~L} \mathrm{ha}^{-1}\left(\mathrm{G} 2\right.$ at $\left.9.2 \mathrm{~km} \mathrm{~h}^{-1}\right)$. It was concluded that for soil mobilization up to $0.3 \mathrm{~m}$ depth, scarification is more advantageous than subsoiling, promoting an economy of $6.6 \mathrm{~L} \mathrm{ha}^{-1}$. It is recommended to use higher speed gears, within the limits allowed for tractor size and operation quality.

Keywords: drawbar pull, fuel consumption, scarification, subsoiling, harrowing.
\end{abstract}

\section{INTRODUÇÃO}

No meio agrícola, há a necessidade de realização de inúmeras operações de campo visando principalmente a produção vegetal. Dentre estas, é prática comum a realização de preparos periódicos do solo, mesmo que a cada 3 ou 4 anos, como no estado de Mato Grosso, onde em virtude das condições climáticas e de solo, utiliza-se de diferentes maneiras de mobilização do solo inerentes aos distintos objetivos. Para Gassen et al. (2014), o aumento do tráfego de máquinas agrícolas de maior tamanho, agrava a compactação do solo, afetando a produtividade das culturas. Para solucionar este problema tem-se realizado a escarificação, contudo, esta operação apresenta elevada demanda de tração. Em complemento aos autores, observa-se ainda que pode-se utilizar o subsolador, o qual trabalha em profundidade maiores (acima de $0,3 \mathrm{~m}$ ) e requer ainda mais esforço de tração.

Quando o objetivo é a incorporação de corretivos e fertilizantes, nivelamento do solo ou outros que necessitam do completo revolvimento do solo na camada vertical (profundidade) em que atuam, comumente utiliza-se de grades de discos. As grades possuem muitas variações de tamanho, variando entre outras características, no diâmetro e número de discos, espaçamento entre discos e massa por disco.

O trator é a principal fonte móvel de potência utilizada para realizar as diversas tarefas necessárias nos processos de produção agrícola (GABRIEL FILHO et al. 2008). A função prioritária dos tratores agrícolas, segundo Márquez (2012), é de exercer esforços de tração nas máquinas que realizam um determinado trabalho agrícola, devendo atender a grande versatilidade demandada pelas operações. Nesta versatilidade inclui-se o seu ajuste aos trabalhos pesados, médios e mais leves, sendo recomendado a adequação para cada caso.

De acordo com Gabriel Filho et al. (2010a), uma das principais funções dos tratores agrícolas é transformar a energia do combustível e fornecê-la através da barra de tração (BT), objetivando o tracionamento de máquinas e implementos. O desempenho na barra de tração, depende de vários fatores, como potência do motor, transmissão, 
distribuição de massa nos rodados, e da condição da superfície do solo. Além destes fatores citados, os autores mencionam ainda, confirmado também por Gabriel Filho et al. (2010b), que em trabalhos com marcha de maior velocidade, estas apresentam-se com melhor desempenho operacional do conjunto. Ainda, em favor da marcha de maior velocidade, verificaram maior potência na barra e menor consumo específico de combustível.

O trator é submetido a atividades em superfícies de pouca aderência geralmente em solos mobilizados ou com cobertura vegetal, sendo que em ambas as condições, na interface solopneu há perdas de potência devido à patinagem dos rodados motrizes (GABRIEL FILHO et al., 2004; CORTEZ et al., 2009). A normalização técnica (OECD, 2012) prevê a limitação da patinagem a 15\%. Acima deste limite há muitas perdas de potência em função da redução da velocidade de avanço do trator (MÁRQUEZ, 2012), além de haver dispêndio energético desnecessário e desgaste prematuro de pneus e demais componentes do trator.

Segundo a ASABE (2011a), para um trator operar com máxima eficiência de tração, a patinagem dos rodados motrizes deve estar entre 8 e $10 \%$ em solos não mobilizados ou firmes, 11 e $13 \%$ em solos mobilizados e entre 14 e $16 \%$ em solos soltos ou arenosos. Estudos realizados por Upadhyaya et al., (1997) e Elwaleed et al., (2006), mostraram que a eficiência trativa apresentou os melhores índices, nas condições de tração em que a patinagem ficou entre 5 e $10 \%$.

Um trator operando sobre superfície de solo firme, segundo a norma D497.7 da ASABE (2011b), apresenta um desempenho com eficiência na barra de tração de aproximadamente $68 \%$. Para solos preparados, essa eficiência cai para $65 \%$ e para solos soltos para $58 \%$.

Gabriel Filho et al. (2004) avaliaram o desempenho operacional de um trator agrícola em operação de escarificação sobre solo agrícola em diferentes condições, incluindo sem cobertura vegetal. Os resultados do experimento mostraram uma eficiência trativa, obtida por modelos matemáticos, na ordem de $72 \%$. Ainda, observaram um esforço médio de $5 \mathrm{kN}$ por haste e potência na barra de tração de $5,14 \mathrm{~kW}$ por haste. Por sua vez, Santos et al. (2011), avaliaram um escarificador em solo argiloso e observaram uma demanda média de tração de $4,46 \mathrm{kN}$ por haste.

Salvador et al. (2008) avaliaram um subsolador antes e depois de diferentes preparos do solo, e verificaram uma demanda média de potência de $7,9 \mathrm{~kW}$ por haste, com subsolagem antes de outros preparos (aração e gradagem) e de $6,7 \mathrm{~kW}$ por haste para subsolagem após os preparos. Respectivamente, os consumos operacionais foram de 26,95 e $22,48 \mathrm{~L} \mathrm{ha}^{-1}$ e com consumos específicos respectivos de $439 \mathrm{e}$ $469 \mathrm{~g} \mathrm{kWh}^{-1}$. Conforme Simões et al. (2011) o item combustível é o principal componente dentre os demais que compõem o custo operacional das máquinas agrícolas durante a operação de subsolagem, impactando diretamente nos custos finais de produção.

Fernandes et al. (2008) realizaram um trabalho comparativo entre preparo convencional, cultivo mínimo ou reduzido e plantio direto. Nos preparos reduzidos com vibro escarificador encontraram consumo de $17,1 \mathrm{~L} \mathrm{ha}^{-1}$ e com grade leve de $14,3 \mathrm{~L} \mathrm{ha}^{-1}$. Os autores concluíram que o cultivo mínimo representa uma economia energética de $22,5 \%$ quando comparado ao preparo convencional.

Miranda (2000), observaram que a subsolagem é uma operação com grande gasto de combustível, sendo que nos resultados encontrados para $0,5 \mathrm{~m}$ de profundidade, o consumo foi da ordem de $27 \mathrm{~L} \mathrm{ha}^{-1}$. Evidenciaram ainda, que é possível economizar 4,9 $\mathrm{L} \mathrm{ha}^{-1}$ de combustível quando operando em marcha de maior velocidade, dentro da capacidade do trator.

O objetivo do trabalho foi avaliar o desempenho de um trator agrícola de $134 \mathrm{~kW}$ operando com quatro equipamentos de preparo de solo com demandas distintas de tração, variando-se marchas de trabalho e buscando resultados que promovessem a melhor recomendação de uso, principalmente no requisito energético.

\section{MATERIAL E MÉTODOS}

O trabalho foi conduzido junto ao Laboratório de Agricultura de Precisão e Mecanização Agrícola (LAPMec) da Universidade Federal de Mato Grosso, campus universitário de Sinop, tendo como localização geográfica central, latitude $11^{\circ} 51^{\prime} 59,55^{\prime}$ 'S, longitude $55^{\circ} 28^{\prime} 50,15^{\prime} \mathrm{N}$ e altitude de $380 \mathrm{~m}$. O solo da área do experimento é do tipo argiloso com granulometria fracionada em $51,2 \%$ de argila, $27,9 \%$ de areia e $20,9 \%$ de silte. Durante os ensaios (novembro de 2017) o mesmo estava com umidade gravimétrica entre 22 e $24 \%$. A condição do solo no momento dos trabalhos estava sem cobertura vegetal, sendo que anteriormente, no final da estação chuvosa em Mato Grosso (abril), foi realizado o preparo convencional com gradagem intermediária seguida de niveladora. Após esse período o solo ficou em pousio.

Para os ensaios utilizou-se de um trator agrícola 4x2 TDA modelo T7.205 da marca New Holland, potência nominal do motor de $134 \mathrm{~kW}$, lastreado e com massa de 99,1 kN (10.100 $\mathrm{kg}$ ), equipado com pneus radiais 520/85 R38 duplos (traseiros) e 420/85 R28 simples na dianteira. Foram utilizados quatro equipamentos para preparo do solo, sendo: Escarificador (Esc.) modelo Fox da marca Stara com 11 hastes espaçadas em $0,3 \mathrm{~m}$ (totalizando 3,3 $\mathrm{m}$ de faixa de trabalho), com ponteiras de $50 \mathrm{~mm}$ de largura e operando a $0,3 \mathrm{~m}$ de profundidade; Subsolador (Sub.) marca KLR de 5 hastes com ponteiras de 80 $\mathrm{mm}$ de largura operando a $0,4 \mathrm{~m}$ de profundidade. $\mathrm{O}$ espaçamento entre hastes era de $0,45 \mathrm{~m}$ totalizando largura de trabalho de 2,25 m. Ambos (Esc. e Sub.) possuíam discos de corte de palha à frente das hastes e rolo destorroador posicionado posteriormente. Utilizou-se ainda de uma grade intermediária (G1-28) de 28 discos com diâmetro de $711 \mathrm{~mm}$ ( 28 x 28"); e uma grade também intermediária (G1-26) com 24 discos de $660 \mathrm{~mm}$ de diâmetro ( 24 x 26"), sendo que ambas as grades possuíam espaçamento entre discos de $270 \mathrm{~mm}$ e eram da marca Tatu Marchesan. Os equipamentos mencionados são de uso comum em áreas produtoras de grãos e fibras do estado de Mato Grosso, para fins de descompactação, nivelamento e condicionamento do perfil do solo, incorporação de corretivos e fertilizantes, e entre outras funções.

Foram instalados no trator diversos sensores e equipamentos para aquisição de dados, permitindo assim a obtenção de parâmetros energéticos e de desempenho nas quatro operações. Sendo assim obteve-se: I - Força na barra de tração do trator; II - Velocidade de deslocamento. III Patinagem dos rodados motrizes; IV - Rotação do motor; V Consumo de combustível; e a partir destes, calculou-se ainda: VI - Potência na barra de tração; VII - Capacidade operacional; VIII - Consumo específico e operacional; IX Eficiência na barra de tração; e X - Eficiência trativa. Os dados coletados eram enviados para um sistema de aquisição de dados via bluetooth programado para esse tipo de ensaio. 
I - Para obtenção da demanda de tração na barra, utilizouse de um suporte adaptável (berço), em estrutura metálica, onde foi fixado uma célula de carga com capacidade máxima $100 \mathrm{kN}$ (Figura 1).

II - A velocidade de deslocamento foi obtida com GPS de uso agrícola devidamente aferido, instalado na parte superior da cabine do trator.

III - A patinagem dos rodados motrizes, foi obtida com os dados do GPS e com encoders incrementais (sensor de pulso) instalados no centro das rodas traseiras e dianteiras dos trator. Utilizou-se o modelo E30-A-1-B de 5 a 28V, marca S\&E de 60 pulsos por volta.

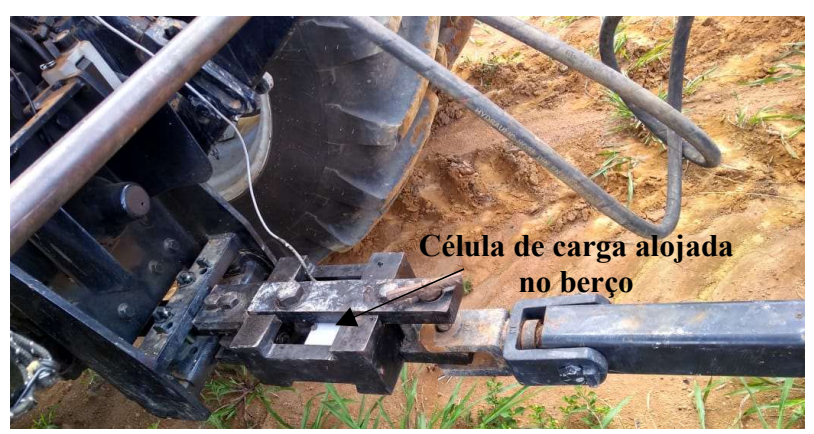

Figura 1. Suporte tipo berço com célula de carga, instalado no trator em substituição a barra de tração original e acoplado ao cabeçalho do subsolador.

Figure 1. Instrumented drawbar mounted on the tractor attached to the subsoiler hitch.

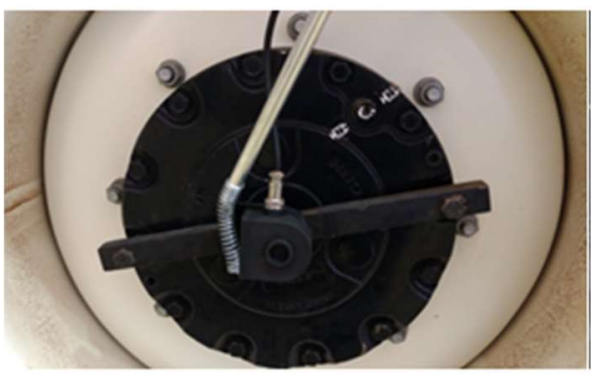

Figura 2. Detalhe de um encoder instalado no centro da roda motriz dianteira.

Figure 2. Detail of the encoder installed in the center of the front wheel drive.

O cálculo da patinagem foi feito conforme Equação 1, adaptada da ASABE (2011a).

$$
\text { Pat }=\frac{\mathrm{An}-\mathrm{A} 1}{\mathrm{An}} .100
$$

(Equação 1)

em que: Pat = patinagem, em \%; An = espaço percorrido obtido pelo GPS, em m; e A1 = espaço teórico, calculado pela circunferência de rolamento e pelo número de voltas das rodas motrizes obtido com encoder, $\mathrm{m}$.

IV - Para obtenção da rotação do motor, também foi utilizado de encoders e a partir da relação de transmissão do trator.

V - Para o consumo de combustível, foram instalados dois fluxômetros no circuito de combustível do motor. O modelo utilizado foi o OVAL M-III LSF41L0-M2, sendo um instalado na entrada de combustível antes do sistema de injeção e outro no retorno para o reservatório de combustível ("tanque"). O aquisitor de dados já estava programado para calcular e armazenar o consumo numa frequência de $1 \mathrm{HZ}$.

VI - De acordo com Zoz; Grisso (2003), o principal ponto a ser observado nos tratores agrícolas é o desempenho na barra de tração, definido pelo produto entre a força desenvolvida e a velocidade de deslocamento (Equação 2).

$$
\mathrm{PBT}=\frac{\mathrm{FT} \cdot \mathrm{V}}{3,6}
$$

em que: $\mathrm{P}_{\mathrm{BT}}=$ potência na barra de tração em $\mathrm{kW} ; \mathrm{FT}=$ Força de tração, em kN; e V = Velocidade de deslocamento, em $\mathrm{km} \mathrm{h}^{-1}$;

VII - A capacidade operacional foi obtida a partir da Equação 3.

$$
\mathrm{CO}=\frac{\mathrm{L} \cdot \mathrm{V}}{10} \cdot \mathrm{Ef}
$$

em que: $\mathrm{CO}=$ Capacidade operacional, em ha $\mathrm{h}^{-1} ; \mathrm{L}=$ largura efetiva de trabalho dos equipamentos de preparo do solo, em $\mathrm{m}$; e $\mathrm{Ef}=\mathrm{a}$ eficiência de campo adotada como $80 \%$.

VIII - O consumo específico e operacional foram obtidos conforme Equação 4 e 5.

$$
\mathrm{Cop}=\frac{\mathrm{Chv}}{\mathrm{CO}}
$$

em que: $\mathrm{COp}=$ consumo operacional, $\mathrm{em} \mathrm{L} \mathrm{ha}^{-1} ; \mathrm{Chv}=$ Consumo horário volumétrico, em $\mathrm{L} \mathrm{h}^{-1}$;

$$
\mathrm{CE}=\frac{\text { Chv. } \rho}{\text { PBT }}
$$

em que: $\mathrm{CE}=$ Consumo específico de combustível, em $\mathrm{g} \mathrm{kWh}^{-1} ; \rho=$ massa específica do combustível, em $\mathrm{kg} \mathrm{L}^{-1}$.

IX - Eficiência na barra de tração, foi calculada conforme equação 6 .

$$
\eta \mathrm{BT}=\frac{\mathrm{PBT}}{\mathrm{P}_{\mathrm{M}}} .100
$$

onde: $\eta=$ Eficiência na barra, \%; e $\mathrm{P}_{\mathrm{M}}=$ Potência nominal do motor fornecido pelo fabricante.

X - A eficiência trativa, descrita por alguns autores como eficiência tratória ou eficiência de tração, é a razão entre a potência de saída na barra de tração e a potência nos eixos motrizes (ZOZ; GRISSO, 2003; TIWARI et al., 2009). A ASABE (2011b), através da norma D497.7, apresenta a Equação 7, na qual utiliza-se a tração bruta (entrada), a tração líquida (saída) e a patinagem.

$$
\mathrm{ET}=(1-\mathrm{Pat}) \cdot \frac{\mathrm{FT}}{\mathrm{TB}}
$$

em que: $\mathrm{ET}=$ Eficiência trativa em decimal; e TB = tração bruta, em kN e calculada pela Equação 8.

$$
\mathrm{TB}=\mathrm{FT}+\mathrm{RR}
$$

em que: $\mathrm{RR}=$ Resistência ao rolamento, em $\mathrm{kN}$, obtido por determinação direta em campo com comboio de dois tratores. 
A aquisição de dados foi feita por uma central de coleta programada especificadamente para ensaios de tratores. A visualização e armazenamento de dados foram feitas em um tablet com programa computacional também desenvolvido especificadamente para este fim.

Além dos sensores, foi utilizado de uma balança com quatro plataformas individuais para fazer a determinação e aferição da massa do trator, distribuindo-se na proporção de $65 \%$ no eixo traseiro e $35 \%$ no eixo dianteiro em um total de $99,1 \mathrm{kN}$.

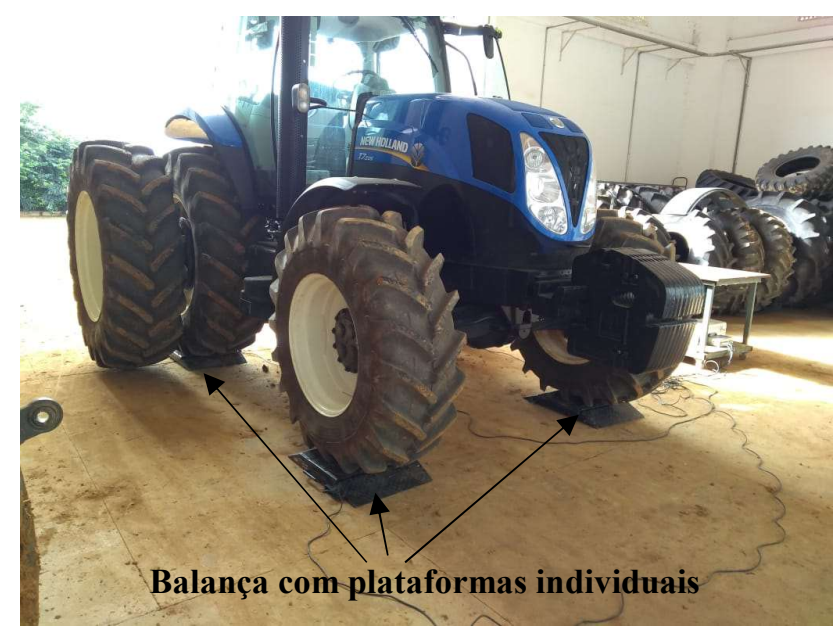

Figura 3. Trator sobre balança com quatro plataformas individuais para determinação e ajuste de massa entre os eixos.

Figure 3. Wheel weighing scale being used for tractor ballasting.

O trabalho foi delineado em blocos casualizados em esquema fatorial, onde foram avaliados, além dos quatro equipamentos, o fator marcha, com duas velocidades de trabalho para cada operação, trabalhando-se com as marchas recomendadas para cada situação, sendo as mais próximas de 5 e $6 \mathrm{~km} \mathrm{~h}^{-1}$ para subsolagem e escarificação, de 6,0 e 7,5 km $\mathrm{h}^{-1}$ para grade $28 \times 28$, e 6,5 e $9,0 \mathrm{~km} \mathrm{~h}^{-1}$ para grade $24 \times 26$. As distintas marchas escolhidas para cada operação, se baseou no maior aproveitamento de potência do trator, no tamanho do equipamento e também na qualidade da operação. Foram realizadas 5 repetições, totalizando 40 unidades experimentais. Os resultados obtidos foram submetidos à análise de variância pelo teste de Tukey a $5 \%$ de probabilidade utilizando o programa SISVAR versão 5.6.

Na Figura 4 observa-se os quatro equipamentos utilizados, sendo dois de hastes e dois de discos.

\section{RESULTADOS}

Observa-se, na Tabela 1, que houve variação significativa do tratamento operação para todos os parâmetros avaliados e para as marchas apenas patinagem e eficiência trativa não foram significativos. A análise da variância para a interação entre os tratamentos, operação e marchas, apresentou diferença significativa para potência na barra de tração, velocidade, rotação do motor, consumo horário volumétrico, eficiência na barra de tração, e capacidade operacional.

$\mathrm{O}$ fato de ter havido significância em todas as operações, tem relação com a demanda de cada uma e que permite variações inerentes a cada condição, mas principalmente a largura de trabalho, a qual permite maior desempenho operacional do conjunto.

A interação para potência na barra de tração, tem relação com a demanda de esforço de cada equipamento e em função das marchas mais rápidas demandarem maior potência do motor já que a velocidade influencia diretamente na potência na barra de tração.

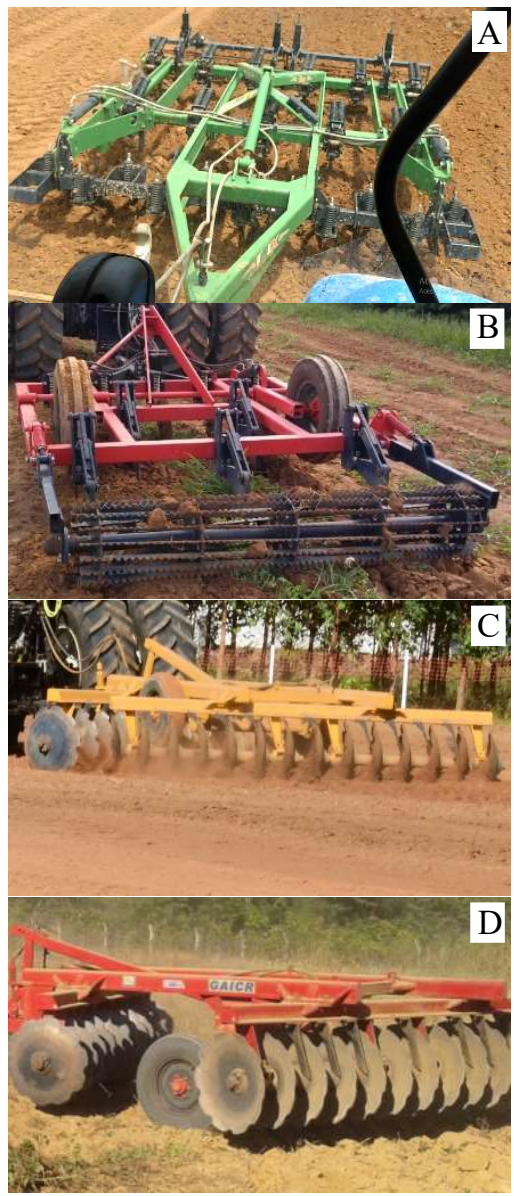

Figura 4. Equipamentos utilizados nos ensaios. (A) escarificador 11 hastes; (B) Subsolador 5 hastes; (C) grade 28x28; (D) e grade 24x26. Figure 4. Equipment used in the tests. (A) scarifier 11 shank; (B) Subsoiler 5 shank; (C) offset disk harrow 28x28; (D) and offset disk harrow $24 \times 26$.

Na Tabela 2 tem-se os valores de interação onde observase, que: para potência na barra de tração, esta diferiu significativamente na grade $24 \times 26$ para a marcha 1 , quando comparado aos demais equipamentos, sendo que o ocorrido se deve principalmente a menor força de tração e uma velocidade que não demandou valores maiores de potência.

Entre as marchas, apenas com subsolador não houve variação dos dados. A explicação se deve a menor variação de velocidade entre as marchas utilizadas $\left(5,1\right.$ e $\left.6 \mathrm{~km} \mathrm{~h}^{-1}\right)$, sendo que nesse caso há uma compensação entre consumo horário e potência produzida, gerando um CE semelhante.

$\mathrm{Na}$ marcha 2 e com o escarificador teve-se a maior potência $(74,5 \mathrm{~kW})$, podendo ser explicado devido a maior demanda de tração associada $(44,9 \mathrm{kN})$, sendo o único a diferir a força entre as marchas; para a força de tração, houve diferença significativa para todas as operações, considerando que são equipamentos e tamanhos distintos. Houve diferença entre marchas apenas para o escarificador, podendo ser atribuído a variação do solo; por fim, ao analisar a velocidade, observa-se diferenças significativas nos quatro equipamentos para a marcha 1, e igual para o subsolador e escarificador na marcha 2. No comparativo entre as marchas, houve significância em todos os tratamentos. 
Tabela 1. Síntese da análise de variância e do teste de médias para as variáveis potência na barra de tração (PBT), força na barra de tração (FT), velocidade (Vel.), rotação do motor (RM), patinagem (Pat.), consumo horário volumétrico (Chv), consumo operacional (Cop), consumo específico (CE), eficiência na barra de tração ( $\eta \mathrm{BT})$, eficiência trativa (ET) e capacidade operacional (CO).

Table 1. Summary of the variance analysis and the test of means for the variables: drawbar power (PBT), drawbar pull (FT), ground speed (Vel.), engine speed (RM), slip (Pat.), volumétric fuel comsumption (Chv), operational consumption (Cop), specific fuel consumption (CE), drawbar efficiency ( $\mathrm{nBT})$, traction efficiency (ET) and operational capacity (CO).

\begin{tabular}{lccccccccccc}
\hline Tratam. & PBT & $\mathrm{FT}$ & $\mathrm{Vel}$ & $\mathrm{RM}$ & Pat. & $\mathrm{Chv}$ & $\mathrm{Cop}$ & $\mathrm{CE}$ & $\eta \mathrm{BT}$ & $\mathrm{ET}$ & $\mathrm{CO}$ \\
\hline Operação & $\mathrm{kW}$ & $\mathrm{kN}$ & $\mathrm{km} \mathrm{h}^{-1}$ & $\mathrm{rpm}$ & $\%$ & $\mathrm{~L} \mathrm{~h}^{-1}$ & $\mathrm{~L} \mathrm{ha}^{-1}$ & $\mathrm{~g} \mathrm{kWh}^{-1}$ & $\%$ & - & $\mathrm{ha} \mathrm{h}^{-1}$ \\
\hline Esc. & $63,6 \mathrm{a}$ & $43,5 \mathrm{a}$ & $5,2 \mathrm{~d}$ & $2231 \mathrm{~b}$ & $10,1 \mathrm{a}$ & $23,3 \mathrm{~b}$ & $16,9 \mathrm{~b}$ & $362 \mathrm{c}$ & $47,5 \mathrm{a}$ & $0,76 \mathrm{a}$ & $1,38 \mathrm{~b}$ \\
Sub. & $58,1 \mathrm{~b}$ & $37,8 \mathrm{~b}$ & $5,5 \mathrm{c}$ & $2247 \mathrm{a}$ & $10,9 \mathrm{a}$ & $23,4 \mathrm{~b}$ & $23,5 \mathrm{a}$ & $396 \mathrm{bc}$ & $43,4 \mathrm{~b}$ & $0,73 \mathrm{~b}$ & $1,00 \mathrm{c}$ \\
G1-28 & $57,9 \mathrm{~b}$ & $30,8 \mathrm{c}$ & $6,8 \mathrm{~b}$ & $2243 \mathrm{a}$ & $8,7 \mathrm{~b}$ & $26,1 \mathrm{a}$ & $13,9 \mathrm{c}$ & $450 \mathrm{a}$ & $43,3 \mathrm{~b}$ & $0,73 \mathrm{~b}$ & $1,89 \mathrm{a}$ \\
G2-26 & $53,4 \mathrm{c}$ & $24,3 \mathrm{~d}$ & $7,9 \mathrm{a}$ & $2243 \mathrm{a}$ & $3,8 \mathrm{c}$ & $23,2 \mathrm{~b}$ & $12,4 \mathrm{~d}$ & $429 \mathrm{ab}$ & $39,9 \mathrm{c}$ & $0,71 \mathrm{c}$ & $1,90 \mathrm{a}$ \\
DMS & 2,63 & 1,88 & 0,09 & 11,28 & 1,13 & 1,98 & 1,34 & 36,40 & 1,97 & 0,01 & 0,025 \\
\hline Marcha & & & & & & & & & & & \\
M1 & $50,4 \mathrm{~b}$ & $33,6 \mathrm{~b}$ & $5,6 \mathrm{~b}$ & $2257 \mathrm{a}$ & $8,4 \mathrm{a}$ & $22,3 \mathrm{~b}$ & $17,4 \mathrm{a}$ & $433 \mathrm{a}$ & $37,6 \mathrm{~b}$ & $0,72 \mathrm{a}$ & $1,35 \mathrm{~b}$ \\
M2 & $66,1 \mathrm{a}$ & $34,7 \mathrm{a}$ & $7,1 \mathrm{a}$ & $2224 \mathrm{~b}$ & $8,4 \mathrm{a}$ & $25,7 \mathrm{a}$ & $16,0 \mathrm{~b}$ & $386 \mathrm{~b}$ & $49,4 \mathrm{a}$ & $0,73 \mathrm{a}$ & $1,73 \mathrm{a}$ \\
DMS & 1,40 & 1,00 & 0,05 & 6,00 & 0,60 & 1,05 & 0,71 & 19,34 & 1,05 & 0,005 & 0,013 \\
\hline Teste F & & & & & & & & & & & \\
Operação & $36,3^{* *}$ & $288,2^{* *}$ & $2197,8^{* *}$ & $5,3^{* *}$ & $117,3^{* *}$ & $7,5^{* *}$ & $200,2^{* *}$ & $16,4 * *$ & $35,9^{* *}$ & $52,1^{* *}$ & $4415,1^{* *}$ \\
Marcha & $525,8^{* *}$ & $5,9^{*}$ & $3675^{* *}$ & $126,4^{* *}$ & $0,0 \mathrm{NS}$ & $45,2 *$ & $16,7 * *$ & $24,4 * *$ & $524,6^{* *}$ & $0,3 \mathrm{NS}$ & $3410,4^{* *}$ \\
Oper.*Marcha & $17,1^{* *}$ & $1,5 \mathrm{NS}$ & $217,8^{* *}$ & $7,3^{* *}$ & $0,9 \mathrm{NS}$ & $3,9^{*}$ & $0,4 \mathrm{NS}$ & $1,4 \mathrm{NS}$ & $16,9^{* *}$ & $0,3 \mathrm{NS}$ & $229,3^{* *}$ \\
\hline CV \% & 3,73 & 4,55 & 1,29 & 0,42 & 11,18 & 6,80 & 6,62 & 7,33 & 3,74 & 1,10 & 1,35 \\
\hline
\end{tabular}

M1 e M2 = Marcha 1 e marcha 2 respectivamente; G28 = grade com 28 discos de 28"; G26 = grade com 24 discos de 26"; NS = Não significativo; *=Significativo a $5 \%$ de probabilidade de erro; $* *=$ Significativo a $1 \%$ de probabilidade de erro; DMS = diferença mínima significativa. Médias seguidas de mesma letra minúscula na coluna, e maiúscula na linha, não diferem estatisticamente (Tukey, $\mathrm{p}>0,05$ ).

Tabela 2. Dados da interação (operação e marcha) para potência na barra de tração (PBT), força de tração (FT) e velocidade de trabalho (Vel.).

Table 2. Interaction data (operation and gear) for drawbar power (PBT), drawbar pull (FT) and ground speed (Vel).

\begin{tabular}{ccccccc} 
Trat. & \multicolumn{3}{c}{ PBT $(\mathrm{kW})$} & \multicolumn{2}{c}{ FT $(\mathrm{kN})$} & \multicolumn{2}{c}{ Vel. $\left(\mathrm{km} \mathrm{h}^{-1}\right)$} \\
\hline \multirow{2}{*}{ Oper } & \multicolumn{5}{c}{ Marchas } \\
& M1 & M2 & M1 & M2 & M1 & M2 \\
\cline { 2 - 7 } Esc. & $52,6 \mathrm{aB}$ & $74,5 \mathrm{aA}$ & $42,0 \mathrm{aB}$ & $44,9 \mathrm{aA}$ & $4,5 \mathrm{~dB}$ & $6,0 \mathrm{cA}$ \\
Sub. & $52,7 \mathrm{aB}$ & $63,5 \mathrm{bA}$ & $37,5 \mathrm{bA}$ & $38,1 \mathrm{bA}$ & $5,1 \mathrm{cB}$ & $6,0 \mathrm{cA}$ \\
G1-28 & $52,3 \mathrm{aB}$ & $63,5 \mathrm{bA}$ & $30,6 \mathrm{cA}$ & $31,1 \mathrm{cA}$ & $6,2 \mathrm{bB}$ & $7,3 \mathrm{bA}$ \\
G2-26 & $43,8 \mathrm{bB}$ & $63,0 \mathrm{bA}$ & $24,1 \mathrm{dA}$ & $24,5 \mathrm{dA}$ & $6,6 \mathrm{aB}$ & $9,2 \mathrm{aA}$ \\
\hline DMS & \multicolumn{2}{c}{3,73} & \multicolumn{2}{c}{2,67} & \multicolumn{2}{c}{0,14} \\
\hline DMS & \multicolumn{2}{c}{2,80} & \multicolumn{2}{c}{2,00} & \multicolumn{2}{c}{0,11} \\
\hline
\end{tabular}

Oper $=$ operação; Esc. $=$ escarificador; Sub. $=$ subsolador; G1-28 $=$ grade de discos 28x28"; G2-26 = grade de discos 24x26".

Observa-se na Tabela 3 que não houve variação de rotação dentro da marcha 1. Na marcha 2, de maiores velocidades e de acordo com cada operação, observa-se que para o escarificador devido a maior força demandada, e a grade $24 \times 26$ que operou a $9,2 \mathrm{~km} \mathrm{~h}^{-1}$, demandaram maior esforço no motor e por consequência houve maior queda de rotação. Comparando a variável rotação dentro de marchas, houve diferenças significativas em todos os tratamentos. Para a patinagem, com a grade $24 \times 26$, por ser mais leve e exigir menor esforço de tração, obteve-se os menores valores, diferindo dos demais. Subsolagem e escarificação obtiveram patinagem mais elevadas tendo apenas a grade $28 \times 28$ que se igualou ao subsolador na marcha 1 . Não houve diferenças significativas entre as marchas.

Verifica-se na Tabela 4, os dados relacionados ao consumo horário volumétrico (Chv) e específico (CE). Na marcha 1 apenas a grade $28 \times 28$ diferiu dos demais. Na marcha 2 os consumos não diferiram estatisticamente. Comparando-se as marchas apenas a grade 28×28 que não teve variância. Já para o consumo específico, o melhor resultado foi com o escarificador, o qual foi significativamente igual ao subsolador. Esse resultado mostra que por serem equipamentos que demandam maior esforço (mesmo que compatíveis com o tamanho do trator) permitiram maior exploração da potência do motor, realizando um melhor aproveitamento energético. Vale ressaltar que, apesar de ter um melhor consumo específico não significa que são operações mais vantajosas para o produtor, sendo que o mesmo deverá observar outros fatores no momento da decisão, como consumo operacional $\left(\mathrm{L} \mathrm{ha}^{-1}\right)$, objetivo do trabalho e outros mais específicos a cada caso.

Tabela 3. Dados da interação entre os tratamentos para a rotação do motor (RM) e patinagem dos rodados (Pat.).

Table 3. Data of the interaction between the treatments for engine speed (RM) and slip (Pat.).

\begin{tabular}{ccccc}
\hline Trat. & \multicolumn{2}{c}{ RM (rpm) } & \multicolumn{2}{c}{ Pat. (\%) } \\
\cline { 2 - 5 } Oper. & \multicolumn{4}{c}{ Marchas } \\
nse. & M1 & \multicolumn{2}{c}{ M1 } & M2 \\
\cline { 2 - 5 } Esc. & $225 \mathrm{aA}$ & $2206 \mathrm{cB}$ & $10,7 \mathrm{aA}$ & $10,4 \mathrm{aA}$ \\
Sub. & $2255 \mathrm{aA}$ & $2238 \mathrm{aB}$ & $9,9 \mathrm{abA}$ & $11,1 \mathrm{aA}$ \\
G1-28 & $2253 \mathrm{aA}$ & $2231 \mathrm{abB}$ & $8,7 \mathrm{bA}$ & $8,6 \mathrm{bA}$ \\
G2-26 & $2264 \mathrm{aA}$ & $2220 \mathrm{cbB}$ & $4,1 \mathrm{cA}$ & $3,4 \mathrm{cA}$ \\
\hline DMS coluna & \multicolumn{2}{c}{15,96} & \multicolumn{2}{c}{1,60} \\
\hline DMS linha & \multicolumn{2}{c}{12,00} & \multicolumn{2}{c}{1,20} \\
\hline
\end{tabular}

Oper $=$ operação; Esc. $=$ escarificador; Sub. $=$ subsolador; G1-28 $=$ grade de discos $28 \times 28 " ;$ G2-26 = grade de discos $24 \times 26 "$ ".

Observa-se na Tabela 5 que houve diferenças significativas tanto no comparativo entre operações quanto entre as marchas para a variável resposta capacidade operacional. Isso ocorre devido a dependência desta variável à largura do equipamento, distinta entre os quatro avaliados, e também em função da velocidade de trabalho. Os maiores valores ficaram para a grade $28 \times 28$ na marcha 1 com 1,73 ha $\mathrm{h}^{-1}$, e $2,22 \mathrm{ha} \mathrm{h}^{-1}$ para a grade $24 \times 26$ na marcha 2 . Este último, se deve ao fato que a velocidade de trabalho foi de $9,2 \mathrm{~km} \mathrm{~h}^{-1}$, podendo-se assim aproveitar melhor a potência de motor e obter maior área trabalhada por hora. A variável consumo 
operacional é tida como uma das mais úteis para o usuário, pois trata-se do gasto efetivo de combustível. O pior consumo foi com subsolador na marcha 1 , com $24,1 \mathrm{~L} \mathrm{ha}^{-1}$ sendo este não diferente estatisticamente na marcha 2. A operação de subsolagem visa trabalhar o perfil vertical do solo, e nesse caso foi de $0,4 \mathrm{~m}$ de profundidade. Assim, tem-se um equipamento com menor largura devido ao menor número de hastes, mas que demanda alta potência devido ao esforço requerido por estes órgãos ativos. A grade $24 \times 26$, que operava com a menor profundidade $(120 \mathrm{~mm})$ teve o menor consumo com $11,6 \mathrm{~L} \mathrm{ha}^{-}$ ${ }^{1}$, semelhante ao da grade $28 \times 28 \mathrm{com} 12,9 \mathrm{~L} \mathrm{ha}^{-1}$, ambos na marcha 2. Reitera-se que para as duas grades, há três fatores que permitiram o menor consumo por área, sendo: menores profundidades de trabalho; maior largura; e maior velocidade de deslocamento. Mais uma vez, vale ressaltar que cabe ao usuário escolher a operação que melhor atende a sua necessidade, sendo que para descompactação do solo, é recomendado equipamentos que trabalham em subsuperfície, indicando-se o escarificador para profundidades de até $0,3 \mathrm{~m}$, no qual pode-se observar um consumo sem diferença estatística entre as marchas, variando de $16,4 \mathrm{~L} \mathrm{ha}^{-1}(\mathrm{M} 2=6$ $\left.\mathrm{km} \mathrm{h}^{-1}\right)$ a $17,4 \mathrm{~L} \mathrm{ha}^{-1}\left(\mathrm{M} 1=4,5 \mathrm{~km} \mathrm{~h}^{-1}\right)$.

Tabela 4. Interação entre os fatores operação e marcha para as variáveis consumo horário volumétrico (Chv) e consumo específico (CE).

Table 4. Interaction between the operation and gear factors for the variables volumetric fuel consumption (Chv) and specific fuel consumption (CE).

\begin{tabular}{ccccc}
\hline Trat. & \multicolumn{2}{c}{ Chv $\left(\mathrm{L} \mathrm{h}^{-1}\right)$} & \multicolumn{3}{c}{$\mathrm{CE}\left(\mathrm{g} \mathrm{kW} \mathrm{h}^{-1}\right)$} \\
\cline { 2 - 5 } Oper. & \multicolumn{4}{c}{ Marchas } \\
ny 1 & \multicolumn{2}{c}{ M2 } & M1 \\
Esc. & $20,7 \mathrm{bB}$ & $25,9 \mathrm{aA}$ & $382 \mathrm{cA}$ & $343 \mathrm{bB}$ \\
Sub. & $21,9 \mathrm{bB}$ & $24,8 \mathrm{aA}$ & $406 \mathrm{bcA}$ & $386 \mathrm{abA}$ \\
G28 & $25,7 \mathrm{aA}$ & $26,5 \mathrm{aA}$ & $487 \mathrm{aA}$ & $413 \mathrm{aB}$ \\
G26 & $20,7 \mathrm{bB}$ & $25,7 \mathrm{aA}$ & $457 \mathrm{abA}$ & $402 \mathrm{aB}$ \\
\hline DMS coluna & \multicolumn{2}{c}{2,80} & \multicolumn{2}{c}{51,48} \\
\hline DMS linha & \multicolumn{2}{c}{2,10} & \multicolumn{3}{c}{38,69}
\end{tabular}

Oper $=$ operação; Esc. $=$ escarificador; Sub. $=$ subsolador; G1-28 = grade de discos 28x28"; G2-26 = grade de discos 24x26".

Tabela 5. Interação entre os fatores operação e marcha para capacidade operacional (CO) e consumo operacional (Cop).

Table 5. Interaction between the operation and gear for operational capacity (CO) and operational consumption (Cop).

\begin{tabular}{ccccc}
\hline Trat. & \multicolumn{3}{c}{$\mathrm{CO}\left(\mathrm{ha} \mathrm{h}^{-1}\right)$} & \multicolumn{3}{c}{ Cop $\left(\mathrm{L} \mathrm{ha}^{-1}\right)$} \\
\cline { 2 - 5 } Oper. & \multicolumn{4}{c}{ Marchas } \\
\cline { 2 - 5 } Esc. & $\mathrm{M} 1,19 \mathrm{cB}$ & $1,58 \mathrm{cA}$ & $17,4 \mathrm{bA}$ & $16,4 \mathrm{bA}$ \\
Sub. & $0,91 \mathrm{~dB}$ & $1,08 \mathrm{dA}$ & $24,1 \mathrm{aA}$ & $22,9 \mathrm{aA}$ \\
G1-28 & $1,73 \mathrm{aB}$ & $2,06 \mathrm{bA}$ & $14,9 \mathrm{cA}$ & $12,9 \mathrm{cB}$ \\
G2-26 & $1,57 \mathrm{bB}$ & $2,22 \mathrm{aA}$ & $13,1 \mathrm{cA}$ & $11,6 \mathrm{cB}$ \\
\hline DMS coluna & \multicolumn{2}{c}{0,036} & \multicolumn{2}{c}{1,89} \\
\hline DMS linha & \multicolumn{2}{c}{0,027} & \multicolumn{2}{c}{1,42} \\
\hline
\end{tabular}

Oper = operação; Esc. $=$ escarificador; Sub. $=$ subsolador; G1-28 $=$ grade de discos 28x28"; G2-26 = grade de discos 24x26".

Na Tabela 6 estão dispostos os dados para eficiência na barra de tração e eficiência trativa. Para a primeira, o menor resultado se deu com a grade $24 \times 26$ na marcha 1, operação mais leve com menor utilização da potência do motor, já que para o cálculo utilizou-se como referência o a potência nominal do motor de $134 \mathrm{~kW}$. Na marcha 2, verificou-se um rendimento de 55,7\% para escarificação, maior valor devido a nesta condição ter demandado maior potência na barra. Houve diferença entre as marchas, já que as mais rápidas foram mais eficientes. A eficiência trativa, que considera 0 aproveitamento de potência após esta chegar aos rodados motrizes, verifica-se que nas duas marchas o melhor valor foi para escarificação. Por outro lado, com a grade $24 \times 26$ teve-se os menores resultados, e neste caso a mais baixa eficiência trativa se deve a lastragem elevada do trator sendo que para esta operação (mais leve) o trator pode ter uma massa menor. Não houve diferença entre as marchas para a eficiência trativa.

Tabela 6. Valores da interação (operação e marcha) para eficiência na barra de tração $(\eta \mathrm{BT})$ e eficiência trativa $(\mathrm{ET})$.

Table 6. Interaction values (operation and gear) for drawbar efficiency $(\eta \mathrm{BT})$ and traction efficiency (ET).

\begin{tabular}{|c|c|c|c|c|}
\hline \multirow{3}{*}{ Oper. } & & $\%)$ & & \\
\hline & \multicolumn{4}{|c|}{ Marchas } \\
\hline & M1 & M2 & M1 & M2 \\
\hline Esc. & $39,2 \mathrm{aB}$ & $55,7 \mathrm{aA}$ & $0,76 \mathrm{aA}$ & $0,76 \mathrm{aA}$ \\
\hline Sub. & $39,4 \mathrm{aB}$ & $47,4 \mathrm{bA}$ & $0,73 \mathrm{bA}$ & $0,73 \mathrm{bA}$ \\
\hline G1-28 & $39,1 \mathrm{aB}$ & $47,4 \mathrm{bA}$ & $0,73 \mathrm{bA}$ & $0,74 \mathrm{bA}$ \\
\hline G2-26 & $32,7 \mathrm{bB}$ & $47,1 \mathrm{bA}$ & $0,71 \mathrm{cA}$ & $0,71 \mathrm{cA}$ \\
\hline DMS coluna & \multicolumn{2}{|c|}{2,80} & \multicolumn{2}{|c|}{0,014} \\
\hline DMS linha & \multicolumn{2}{|c|}{2,10} & \multicolumn{2}{|c|}{0,010} \\
\hline
\end{tabular}

Oper = operação; Esc. $=$ escarificador; Sub. $=$ subsolador; G1-28 $=$ grade de discos 28x28"; G2-26 = grade de discos 24x26".

\section{DISCUSSÃO}

A força de tração foi diferente estatisticamente entre todos os equipamentos e semelhante entre as machas, exceto para o escarificador que teve variação entre 42 (M1) e 44,9 kN (M2). Fazendo-se uma análise específica, tem-se demandas entre 3,8 e $4,1 \mathrm{kN}$ por haste, valor que se aproxima dos obtidos por Santos el al. (2011) com 4,6 kN por haste. Comparando-se com Gabriel Filho et al. (2004), tem-se um maior distanciamento de resultados, já que os autores obtiveram demanda unitária de escarificação igual a $5 \mathrm{kN}$. Observou-se que na marcha de maior velocidade, em todas as operações, houve maior potência. Os resultados corroboram com Gabriel Filho et al. (2010a e 2010b). Na escarificação obteve-se na marcha 2 (6 $\left.\mathrm{km} \mathrm{h}^{-1}\right)$ a maior potência $(74,5 \mathrm{~kW})$. A potência específica na escarificação ficou entre 4,8 e $6,8 \mathrm{~kW}$ por haste, aproximandose de 5,1 kW encontrado por Gabriel Filho et al. (2004). Na subsolagem, os valores encontrados variaram de 10,5 a 12,7 $\mathrm{kW}$ haste, ficando distante do valor evidenciado por Salvador et al. (2008), igual a 7,9 kW haste. A grade 24×26, como já era esperado devido ao seu menor tamanho, teve o menor requerimento de força trativa com média de $24,3 \mathrm{kN}$. Conseguiu-se equiparar as potências na marcha 2, para subsolador, grade $28 \times 28$ e grade $24 \times 26$, sendo possível em função do aumento da velocidade a medida que o equipamento demandara menor esforço de tração. Conforme cita Zoz; Grisso (2003), o desempenho na barra de tração é defenido pelo produto entre a força e a velocidade de deslocamento, e neste caso foi possível compensar, destacando-se a G2 (24×26) com $9,2 \mathrm{~km} \mathrm{~h}^{-1}$.

A rotação do motor, observa-se a significativa diferença entre as marchas, explicado em virtude de exigir mais do motor quando o trator se desloca mais rapidamente, havendo então queda de rotação para busca de torque e potência. A patinagem foi menor nas operações que exigiram menos força, destacando-se as duas grades. Cabe ressaltar que a G2 (24x26) o índice de patinagem médio foi de $3,8 \%$ e conforme 
recomenda a ASABE (2011a) este valor está abaixo das recomendações, que variam de 8 a 10 para solos mais firmes, como era o caso do experimento. Neste caso recomenda-se reduzir a lastragem do trator, retirando-se água dos pneus e lastros metálicos. Nos demais preparos, a lastragem está adequada ultrapassando apenas cerca de $1 \%$ do limite máximo, sendo, portanto, tolerável.

No que tange ao consumo de combustível, da-se atenção preferencial ao específico e operacional, sendo este segundo ainda mais útil ao usuário. Com exceção a da gradagem $28 \times 28$, observou-se igualdade do consumo específico entre as marchas, sendo este comportamento usualmente encontrado na literatura (Gabriel Filho et al., 2010a e 2010b). Os menores consumos operacionais, foram obtidos nas operações de gradagem, em que em ambas houve diferenciação entre as marchas, não sendo encontrado o mesmo comportamento com subsolador e escarificador. A média de consumo do trator na M2 com as duas grades, foi de 12,3 $\mathrm{L} \mathrm{ha}^{-1}$, valor abaixo do encontrado por Fernandes et al. (2008) com 14,3 L ha-1. Comparando-se os equipamentos de hastes, ambos diferiram entre si, mas não entre as marchas. Por serem equipamentos que exigiram mais do trator, a variação de velocidade entre as duas marchas utilizadas, não foi suficiente para melhorar o rendimento do combustível por área. Na escarificação a média foi de 16,9 $\mathrm{L} \mathrm{ha}^{-1}$, concordando com Fernandes et al. (2008). Já na subsolagem, verificou-se uma média de $23,5 \mathrm{~L} \mathrm{ha}^{-1}$, próximo dos dados de Salvador et al. (2008) e distanciando-se em 3,5 L quando comparado aos resultados de Miranda (2000), que obteve $27 \mathrm{~L} \mathrm{ha}^{-1}$.

A eficiência na barra de tração foi significativa quando comparando marchas, sendo que o valor maior de $55,7 \%$ foi obtido com escarificador. A ASABE (2011b) prevê que a eficiência pode ser de até $68 \%$ para a condição de solo estudada. Não se atingiu maior eficiência na BT em função de não ter trabalhado com uma terceira marcha, principalmente com escarificador e grade $28 \times 28$, operações que ainda podiam ser exploradas em maiores velocidades das que foram utilizadas, sem perder a qualidade da operação. Para eficiência trativa não houve variação entre marchas, mas obteve-se resultados melhores, proporcionalmente às operações que exploraram melhor a potência do motor, começando por escarificação com 0,76 e patinagem de $10,6 \%$, seguido de subsolagem e gradagem $28 \times 28$ com 0,73 de eficiência em tração e patinagens respectivas de 10,5 e $8,7 \%$. Os resultados vão de encontro com dados de Upadhyaya et al., (1997) e Elwaleed et al., (2006) os quais mencionam que as melhores eficiências são encontradas entre 5 e $10 \%$ de patinagem. São comparáveis ainda a ASABE (2011a), que menciona haver os melhores índices de eficiência trativa, nas condições com margem de 8 a $10 \%$ de patinagem. Com a grade $24 \times 26$, a média de patinagem foi de $3,8 \%$, considerado baixo. Neste caso, há excesso de lastro no trator e assim a eficiência ficou reduzida devido ao maior gasto de potência para transportar massa inutilizada (água nos pneus e lastros metálicos), já que se trata de um equipamento mais leve e com menor demanda de tração.

\section{CONCLUSÕES}

A maior demanda de tração foi obtida com escarificador 11 hastes, seguido de subsolador 5 hastes, grade $28 \times 28$ e grade $24 \times 26$.

Com a grade mais leve $(24 \times 26)$, foi possível equiparar a potência na barra de tração ao subsolador e a grade $28 \times 28$, operando a 9,2 $\mathrm{km} \mathrm{h}^{-1}$, ou seja, a menor demanda de tração nesta, pode ser compensada com maior velocidade de deslocamento.

Recomenda-se para as quatro operações, utilizar as marchas de maior velocidade, já que houve melhor desempenho operacional e energético nestas condições. Ainda poderia ser utilizada uma terceira marcha, tomando-se o cuidado em não prejudicar a qualidade de trabalho e não sobrecarregar o trator.

Para mobilização do solo a até $0,3 \mathrm{~m}$ de profundidade, a escarificação é a melhor opção de preparo, a qual apresentou consumo operacional de combustível intermediário, ficando em 16,9 $\mathrm{L} \mathrm{ha}^{-1}$.

\section{AGRADECIMENTOS}

À Universidade Federal de Mato Grosso - UFMT Campus Sinop, pela infraestrutura concedida e aos colegas do Laboratório de Agricultura de Precisão e Mecanização Agrícola - LAPMec pela ajuda nos trabalhos conduzidos.

\section{REFERÊNCIAS}

ASABE_AMERICAN SOCIETY OF AGRICULTURAL AND BIOLOGICAL ENGINEERS. ASAE EP496.3: agricultural machinery management. St. Joseph: ASABE Standards, 2011a. 7 p.

ASABE_AMERICAN SOCIETY OF AGRICULTURAL AND BIOLOGICAL ENGINEERS. ASAE D497.7: agricultural machinery management data. St. Joseph: ASABE Standards, 2011b. 8 p.

CORTEZ, J. W.; FURLANI, C. E. A.; VIGNA, G. P.; BORSATTO, E. A.; SILVA, R. P. da. Desempenho do trator agrícola no manejo da cultura de cobertura e pressão de inflação do pneu da semeadora. Engenharia Agrícola, Jaboticabal, v. 29, n. 1, p. 72-80, jan./mar. 2009. DOI. http://dx.doi.org/10.1590/S0100-69162009000100008

ELWALEED, A. K.; YAHYA, A.; ZPJADOE, M.; AHMAD, D.; KHEIRALLA, A. F. Net traction ratio prediction for high-lug agricultural tyre. Journal of Terramechanics, Oxford, v. 43, p. 119-139, 2006. DOI: https://dx.doi.org/10.1016/j.jterra.2004.10.002

FERNANDES, H. C.; SILVEIRA, J. C. M.; RINALDI, P. C. N. Avaliação do custo energético de diferentes operações agrícolas mecanizadas. Ciência e Agrotecnologia., Lavras, v. 32, n. 5, p. 1582-1587, set./out. 2008. DOI. http://dx.doi.org/10.1590/S1413-70542008000500034

GABRIEL FILHO, A.; SILVA, S. de L.; MODOLO A. J.; SILVEIRA, J. C. M. da. Desempenho de um trator operando em solo com diferentes tipos de cobertura vegetal. Engenharia Agrícola, Jaboticabal, v. 24, n. 3, p. 781-789, set./dez 2004. http://dx.doi.org/10.1590/S0100-69162004000300032

GABRIEL FILHO, A.; LANÇAS, K. P.; GUERRA, S. P.; PAULA, C. A.; MONTEIRO, L. A. UMEB - Unidade Móvel Para Ensaio da Barra de Tração. Engenharia Agrícola, Jaboticabal, v. 28, n. 4, p. 782-789, 2008. DOI: http://dx.doi.org/10.1590/S0100-69162008000400018

GABRIEL FILHO, A.; LANÇAS, K. P.; LEITE, F.; ACOSTA, J. J. B.; JESUINO, P. R. Desempenho de trator agrícola em três superfícies de solo e quatro velocidades de deslocamento. Revista Brasileira de Engenharia Agrícola e Ambiental, Campina Grande, v.14, n.3, p.333339, 2010a. DOI: http://dx.doi.org/10.1590/S141543662010000300015 
GABRIEL FILHO, A.; MONTEIRO, L. A.; LANÇAS, K. P.; GUERRA, S. P. S.; JESUINO, P. R. Influência da altura das garras dos pneus de um trator em área de plantio direto. Revista Brasileira de Engenharia Agrícola e Ambiental, Campina Grande, v. 14, n. 10, p. 1123-1128, 2010b. DOI: http://dx.doi.org/10.1590/S141543662010001000015

GASSEN, J. R. F.; ALONÇO, A. S.; BAUMHARDT, U. B.; BELLÉ, M. P.; BONOTTO, G. J. Resistência específica à tração na operação de escarificação do solo em camadas de forma simultânea. Revista Brasileira de Engenharia Agrícola e Ambiental, Campina Grande, v.18, n.1, p.116124, 2014. DOI: http://dx.doi.org/10.1590/S141543662014000100015

MÁRQUEZ, L. Tractores Agrícolas: Tecnologias y utilización. Madrid: B\&h Editores, 2012. 844 p.

MIRANDA, N. O.; OLIVEIRA, M.; NUNES, R. L. Desempenho operacional de trator com tração dianteira auxiliar na subsolagem de Um inceptisol. Revista Brasileira de Engenharia Agrícola e Ambiental, Campina Grande, v. 4, n. 1, p. 97-102, 2000. DOI: http://dx.doi.org/10.1590/S1415-43662000000100018

OECD_ORGANISATION FOR ECONOMIC COOPERATION AND DEVELOPMENT. Code 2: OECD standard code for the official testing of agricultural and forestry tractor performance. $2012.90 \mathrm{p}$.

SALVADOR, N.; BENEZ, S. H.; MION, R. L. Consumo de combustível na operação de subsolagem realizada antes e depois de diferentes sistemas de preparo periódico do solo. Engenharia agrícola, Jaboticabal, v. 28, n. 2, p.256-262, abr./jun. 2008. DOI: http://dx.doi.org/10.1590/S010069162008000200006

SANTOS, P. M.; SCHLOSSER, J. F.; RUSSINI, A.; CASALI, A. L.; RIBAS, R. L.; ROBERTI JÚNIOR, O. L. Validação de teorias de simulação para operações com escarificadore em solo argiloso no planalto do Estado do Rio Grande do Sul, Brasil. Ciência Rural, Santa Maria, v. 41, n.1, p. 120 126, jan. 2011. DOI: http://dx.doi.org/10.1590/S010384782011000100019

SIMÕES, D.; SILVA, M. R.; FENNER, P. T. Desempenho operacional e custos da operação de subolagem em área de implantação de eucalipto. Bioscience Journal, Uberlândia, v. 27, n. 5, p. 692-700, 2011.

TIWARI, V. K.; PANDEY, K. P.; SHARMA, A. K Development of a tyre traction testing facility. Journal of Terramechanics, Oxford, n. 46, p. 293-298, 2009. DOI: https://dx.doi.org/10.1016/j.jterra.2009.05.004

UPADHYAYA, S. K.; SIME, M.; RAGHUWANSHI, N.; ADLER, B. Semi-empirical traction prediction equations based on relevant soil parameters. Journal of Terramechanics, Oxford, v. 34, n. 3, p. 141-154, 1997. DOI: https://dx.doi.org/10.1016/S0022-4898(97)00030-X

ZOZ, F. M.; GRISSO, R. D. Traction and tractor performance. Louisville: ASAE, 2003. 46 p. 